\title{
ALMOST CONTINUOUS ORBIT EQUIVALENCE FOR NON-SINGULAR HOMEOMORPHISMS
}

\author{
Alexandre I. Danilenko And Andrés Del Junco
}

\begin{abstract}
Let $X$ and $Y$ be Polish spaces with non-atomic Borel measures $\mu$ and $\nu$ of full support. Suppose that $T$ and $S$ are ergodic non-singular homeomorphisms of $(X, \mu)$ and $(Y, \nu)$ with continuous Radon-Nikodym derivatives. Suppose that either they are both of type $I I I_{1}$ or that they are both of type $I I I_{\lambda}, 0<\lambda<1$ and, in the $I I I_{\lambda}$ case, suppose in addition that both 'topological asymptotic ranges' (defined in the article) are $\log \lambda \cdot \mathbb{Z}$. Then there exist invariant dense $G_{\delta}$-subsets $X^{\prime} \subset X$ and $Y^{\prime} \subset Y$ of full measure and a non-singular homeomorphism $\phi: X^{\prime} \rightarrow Y^{\prime}$ which is an orbit equivalence between $\left.T\right|_{X^{\prime}}$ and $\left.S\right|_{Y^{\prime}}$, that is $\phi\left\{T^{i} x\right\}=\left\{S^{i} x\right\}$ for all $x \in X^{\prime}$. Moreover the Radon-Nikodym derivative $d \nu \circ \phi / d \mu$ is continuous on $X^{\prime}$ and, letting $S^{\prime}=\phi^{-1} S \phi$ we have $T x=S^{\prime n(x)} x$ and $S^{\prime}=T^{m(x)} x$ where $n$ and $m$ are continuous on $X^{\prime}$.
\end{abstract}

\section{INTRODUCTION}

The study of measure-theoretic orbit equivalence was initiated by H. Dye [Dy1], [Dy2] who proved that the orbit equivalence relations of any two ergodic finite measure preserving transformations are measure theoretically isomorphic when restricted to some invariant subsets of full measure. W. Krieger obtained in [Kr1], [Kr2] a far-reaching generalization of Dye's theorem and classified, up to measuretheoretic orbit equivalence, all ergodic non-singular transformations. Dye's and Krieger's theorems extend to ergodic actions of countable amenable groups [CFW]. In contrast to that, any non-amenable countable group has uncountably many nonorbit equivalent probability preserving ergodic actions $[\mathrm{Hj}],[\mathrm{Ep}]$. On the other hand, amenability plays no role in generic orbit equivalence: given any two topologically transitive countable groups of homeomorphisms of perfect Polish spaces, their orbit equivalence relations are homeomorphic after restriction to some invariant dense $G_{\delta}$ subsets [SWWr].

Consider now the class of homeomorphisms of Polish spaces equipped with ergodic nonsingular measures of full support such that the Jacobian (Radon-Nikodym derivative) of the homeomorphism with respect to its non-singular measure is continuous. We consider a notion of orbit equivalence for these which combines the notions of generic and measure-theoretic orbit equivalence. We say that two such homeomorphisms are almost continuously orbit equivalent if their orbit equivalence relations restricted to some invariant dense $G_{\delta}$-subsets of full measure are isomorphic by a nonsingular homeomorphism which has a continuous Radon-Nikodym

1991 Mathematics Subject Classification. 37A.

The first named author thanks the University of Toronto for supporting his visit to Toronto, where the main part of this work was done. 
derivative and such that the co-cycles associated to the orbit equivalence are continuous (see Definition 1.1 below).

T. Hamachi and M. Keane initiated investigation of such a kind of equivalence in the measure-preserving case [HK] by constructing a weakly continuous (finitary in their terminology) orbit equivalence between the binary and ternary odometers. We use the term 'weakly' here since they only required that the orbit equivalence be a homeomorphism between subsets of full measure, although their method in fact does seem to yield a $G_{\delta}$. It is worth mentioning that in the case of transformations defined on infinite product spaces 'weakly almost continuous' is equivalent to 'finitary' in the sense of the celebrated work of M. Keane and M. Smorodinsky [KS]. In a series of subsequent works this was extended to all odometers [R1], irrational rotations [R2] and Bratteli-Vershik adic transformation [HKR] and irreducible Markov chains $[\mathrm{RRu}]$. All of these transformations are probability preserving homeomorphisms of compact metric spaces.

Recently A. del Junco and A. Sahin [dJS] established an almost continuous version of Dye's theorem: any two ergodic probability preserving homeomorphisms of Polish spaces are almost continuously orbit equivalent. The same is true for any ergodic homeomorphisms preserving infinite $\sigma$-finite local measures [dJS]. The purpose of the present paper is to prove an almost continuous version of Krieger's theorem for systems of type $I I I_{\lambda}, 0<\lambda \leq 1$.

If we only require an orbit equivalence to be a homeomorphism between invariant sets of full measure then we obtain a weaker notion of orbit equivalence which we have referred to above here as weakly continuous orbit equivalence. In fact the main result in [JRW] shows that, under mild assumptions, this is no stronger than measure-theoretic orbit equivalence. Although the result there is stated and proved only in the measure-preserving case the statement and its proof remain valid in the non-singular setting. In fact one has the following quite general result.

Theorem. Suppose $X$ and $X^{\prime}$ are separable metric spaces with Borel probability measures $\mu$ and $\mu^{\prime}$ and $G, G^{\prime}$ are countable ergodic groups of measurable nonsingular transformations on $X$ and $X^{\prime}$. If $\phi: X \rightarrow X^{\prime}$ is any measurable nonsingular orbit equivalence of $G, G^{\prime}$ then there exist elements $\sigma, \tau$ in the measurable full groups of $G, G^{\prime}$ such that $\tau \phi \sigma$ (which is also an orbit equivalence!) is a homeomorphism between invariant subsets $X_{0}^{\prime}, Y_{0}^{\prime}$ of full measure.

Section 1 contains the main definitions. We introduce there a topological analogue $r_{\text {top }}$ of the (measure theoretical) asymptotic range for the Radon-Nikodym cocycle of a system. It is a closed subgroup of $\mathbb{R}$ which contains the asymptotic range and it is invariant under almost continuous orbit equivalence. We give an example of type $I I I$ homeomorphisms which are measure-theoretically orbit equivalent but not almost continuously orbit equivalent. In Sections 2 and 3 we prove the the main result of the paper:

Theorem 0.1. Let $(X, \tau, \mu, T)$ and $\left(X^{\prime}, \tau^{\prime}, \mu^{\prime}, T^{\prime}\right)$ be ergodic non-singular homeomorphisms of Polish spaces. If the two systems are either

(i) of type $I I I_{\lambda}, 0<\lambda<1$, and $r_{\text {top }}\left(\rho_{\mu}\right)=r_{\text {top }}\left(\rho_{\mu^{\prime}}\right)=\log \lambda \cdot \mathbb{Z}$, or

(ii) of type $I I I_{1}$

then they are almost continuously orbit equivalent.

In the proof we combine techniques developed in [dJS] for measure-preserving continuous systems with an approach to Krieger's theorem from [KaW] (see also 
[HO]). We also achieve a significant technical simplification of the method of [dJS] by mapping the two spaces involved into a common symbolic space, thereby removing the need for the many-to-one set mappings in [dJS]. We do not know whether the assumption $r_{\text {top }}\left(\rho_{\mu}\right)=r_{\text {top }}\left(\rho_{\mu}^{\prime}\right)=\log \lambda \cdot \mathbb{Z}$ in (i) can be weakened to $r_{\text {top }}\left(\rho_{\mu}\right)=r_{\text {top }}\left(\rho_{\mu}^{\prime}\right)$.

\section{NON-SINGULAR HOMEOMORPHISMS AND GROUPS OF HOMEOMORPHISMS OF POLISH SPACES}

By a dynamical system we mean a quadruple $(X, \tau, \mu, T)$, where $(X, \tau)$ is a Polish space, $\mu$ a non-atomic Borel probability measure on $X$ and $T$ a $\mu$-non-singular homeomorphism of $X$ satisfying

(•) $\mu$ is of full support, i.e. $\mu(O)>0$ for all $O \in \tau$,

(•) $T$ is ergodic and

$(\bullet)$ the Radon-Nikodym derivative $X \ni x \mapsto(d \mu \circ T / d \mu)(x) \in \mathbb{R}$ is continuous (i.e. has a continuous version).

It follows from these assumptions that any set of full measure is dense in $X$, that $X$ is perfect and that $T$ is topologicaly transitive, i.e. there exists a point of $X$ whose orbit is dense. The continuous Radon-Nikodym derivative is defined everywhere in a unique way. We denote the $T$-orbit equivalence relation by $\mathcal{R}_{T}$. It is a Borel subset of $X \times X$. Let $\rho_{\mu}: \mathcal{R}_{T} \rightarrow \mathbb{R}$ stand for the (logarithm of the) Radon-Nikodym cocycle of $T$, i.e. $\rho_{\mu}\left(x, T^{n} x\right)=\log \frac{d \mu \circ T^{n}}{d \mu}(x)$.

Definition 1.1. An invariant dense $G_{\delta}$-subset of full measure (and the restriction of the system to this subset) is called an inessential reduction of the system.

Two systems $(X, \tau, \mu, T)$ and $(Y, \sigma, \nu, S)$ are almost continuously orbit equivalent if there are inessential reductions $X_{0} \subset X$ and $Y_{0} \subset Y$ and a homeomorphism $\phi: X_{0} \rightarrow Y_{0}$ such that

(a) $\phi\left(\left\{T^{n} x: n \in \mathbb{Z}\right\}\right)=\left\{S^{n} \phi x: n \in \mathbb{Z}\right\}$ for all $x \in X_{0}$,

(b) $\mu \circ \phi^{-1} \sim \nu$ and the Radon-Nikodym derivative

$$
Y_{0} \ni y \mapsto\left(d \mu \circ \phi^{-1} / d \nu\right)(y) \in \mathbb{R}
$$

is (i.e. can be chosen) continuous,

(c) letting $S^{\prime}=\phi^{-1} S \phi$ we have $T x=S^{\prime n(x)} x$ and $S^{\prime}=T^{m(x)} x$ where $n$ and $m$ are continuous on $X_{0}$.

Notice that an inessential reduction of a dynamical system is a dynamical system, i.e. the three already mentioned properties marked with $(\bullet)$ are satisfied. Since in this paper we study dynamical systems up to almost continuous orbit equivalence we will often ignore the difference between a dynamical system and an inessential reduction of it and thus use the same notation for them. In particular, as was shown in [dJS], by passing to an invariant dense $G_{\delta}$ subset of full measure we can and shall assume without loss of generality that

$(\bullet)(X, \tau)$ is 0 -dimensional, i.e. there exists a countable base of $X$ consisting of clopen subsets.

In the proof of Theorem 0.1 we will establish that the two systems are almost continuously orbit equivalent to a common intermediate symbolic system where the action is not by a single homeomorphism but by a group of homeomorphims 
so we need to make a few remarks about almost continuous orbit equivalence in this more general context. Suppose $G$ and $H$ are countable groups of non-singular homeomorphisms of Polish probability spaces $X$ and $Y$. Note that we do not assume that $G$ or $H$ acts freely. Definition 1.1 of almost continuous orbit equivalence extends in an obvious way to this context. Condition (c) in that defintion is replaced by the following: letting $G^{\prime}=\phi^{-1} H \phi$, for each $h \in G^{\prime}$ we have $h x=a(x) x$ where $a: X_{0} \rightarrow G$ is continous on $X_{0}$ and for each $g \in G$ we have $g x=b(x) x$ where $b: X_{0} \rightarrow G^{\prime}$ is continuous on $X_{0}$. (Since we have not assumed freeness the functions $a$ and $b$ here need not be uniquely defined.) It is clear that the composition of almost continuous orbit equivalences is again an almost continuous orbit equivalence, after restriction to a suitable inessential reduction.

If $X$ is a Polish probability space and $P(x)$ is a property of a point $x \in X$ then we will say that $P(x)$ holds virtually everywhere if the set where it holds contains a dense $G_{\delta}$ subset of full measure. Any such set will be called virtually full. A function on $X$ will be called virtually continuous if there is a virtually full subset where the restriction of the function is continuous. A subset $E$ is virtually open (closed) if it agrees virtually everywhere with some open (closed) set and virtually clopen if it is virtually closed and open. Equivalently, $E$ is virtually clopen if there is a virtually full subset $X^{\prime}$ such that $E \cap X^{\prime}$ is relatively clopen in $X^{\prime}$, or $1_{E}$ is virtually continuous. Since every virtually full set contains an inessential reduction and we can freely restrict to any inessential reduction we will often blur the distinction between clopen sets and virtually clopen sets, as well as between continuous and virtually continuous functions.

It is also useful to view almost continuous orbit equivalence in terms of a suitable full group. Suppose $G$ is a countable group of non-singular homeomorphisms of a Polish probability space $X$. We consider all homeomorphisms $h: X_{0} \rightarrow X_{1}$ between virtually full subsets $X_{0}$ and $X_{1}$ having the form $h(x)=a(x) x$, where $a: X_{0} \rightarrow G$ is continuous on $X_{0}$. Note that any such $h$ is necessarily non-singular, with a continuous Jacobian. We consider two such homeomorphisms to be equivalent if they agree virtually everywhere.

The collection of equivalence classes is then a group which we will denote by $[G]_{\text {top }}$ and refer to as the topological full group of $G$. Evidently if $\phi$ is an almost continuous orbit equivalence of groups $G_{1}$ and $G_{2}$ then $\phi^{-1}\left[G_{2}\right]_{\text {top }} \phi=\left[G_{1}\right]_{\text {top }}$. Conversely it is easy to check that if $\phi: X_{0} \rightarrow Y_{0}$ is any non-singular homeomorphism between virtually full subsets (invariant or not) which conjugates the topological full groups then (after restriction to a suitable inessential reduction) $\phi$ is an almost continuous orbit equivalence. (The proof of this is formally the same as the proof of the corresponding fact in measure-theoretic orbit equivalence.) Finally we remark that the groups $G_{i}$ need not consist of everywhere defined homeomorphisms: it suffices to have virtually everywhere defined homeomorphisms whose equivalence classes form a group.

We will also have occasion to use the topological full groupoid $[[G]]_{\text {top }}$ of $G$. We define $[[G]]_{\text {top }}$ as the set of all (equivalence classes) of homeomorphisms $h: A \rightarrow B$ such that $A$ and $B$ are virtually clopen subsets of $X$ and $h(x)=a(x) x$ for some $a: A \rightarrow G$ which is continuous on $A$.

The following lemma from [dJS] will be used often in this work.

Lemma 1.2. Let $A \subset X$ be a clopen subset and $\mu(A)=\sum_{i=1}^{\infty} a_{i}$ with $a_{i}>0$. Then there exists a partition $A=\bigsqcup_{i=1}^{\infty} A_{i}$ of $A$ into clopen subsets $A_{i}$ (on an inessential 
reduction) such that $\mu\left(A_{i}\right)=a_{i}$ for all $i$.

Proof. This is essentially Lemma 2(b) of [dJS] where the $A_{i}$ are only open. We may take them to be clopen here because of the implicit inessential reduction. In [dJS] Lemma 2(b) follows from Lemma 2(a) which asserts that for any $\epsilon>0 \mathrm{~A}$ may be partitioned into finitely many clopen sets of measure less than $\epsilon$ and was proved using the (measure-preserving) dynamics. However it is easy to give a nondynamical proof. Let $\left\{U_{i}\right\}$ be a countable basis for the topology and every $U_{i}$ is clopen. Then for each $x \in A$ there is a $U_{i}$ of measure less than $\epsilon$ which contains $x$. Disjointifying the countably many sets so obtained we get a countable partition of $A$ into clopen sets of measure less than $\epsilon$ and the existence of a finite partition follows immediately.

We recall the concept of the asymptotic range $r\left(\rho_{\mu}\right) \subset \mathbb{R}$ of $\rho_{\mu}([\mathrm{Kr} 2],[\mathrm{HO}])$ and define a topological counterpart $r_{\text {top }}\left(\rho_{\mu}\right)$ of this concept.

\section{Definition 1.3.}

(i) A real $t$ belongs to $r\left(\rho_{\mu}\right)$ if for any $\epsilon>0$ and any subset $A \subset X$ of positive measure there are $k \neq 0$ and a subset $B \subset A$ of positive measure such that $\left|\rho_{\mu}\left(x, T^{k} x\right)-t\right|<\epsilon$ for all $x \in B$.

(ii) If the above property holds only for all virtually clopen subsets $A$ of positive measure we write $t \in r_{\text {top }}\left(\rho_{\mu}\right)$. Notice that then the corresponding subset $B$ may be chosen virtually clopen.

It is easy to verify that $r_{\text {top }}\left(\rho_{\mu}\right)$ is a closed subgroup of $\mathbb{R}$ and evidently $r_{\text {top }}\left(\rho_{\mu}\right) \supset$ $r\left(\rho_{\mu}\right)$. It is also easy to verify that the asymptotic range and the topological asymptotic range of the Radon-Nikodym cocycle are invariant under measure-theoretic orbit equivalence and almost continuous orbit equivalence respectively.

Given a subset $A$ of positive measure, $T$ induces a non-singular transformation $T_{A}$ on the space $\left(A, \mu\lceil A)\right.$. If $A$ is clopen then $T_{A} \in[[T]]_{\text {top }}$ and there exists an inessential reduction $X^{\prime}$ of $X$ such that $T_{A \cap X^{\prime}}$ is a homeomorphism of $A \cap X^{\prime}$. In what follows by $T_{A}$ we mean $T_{A \cap X^{\prime}}$. Of course, the $T_{A^{-} \text {-orbit equivalence relation }}$ coincides with $\mathcal{R}_{T} \cap\left(X^{\prime} \cap A \times X^{\prime} \cap A\right)$.

Lemma 1.4. Let $A$ and $B$ be two non-empty clopen subsets of $X$. If one of the following is satisfied:

(i) $T$ preserves $\mu$ and $\mu(A)=\mu(B)$ or

(ii) $T$ is of type III

then there exist open subsets $A^{\prime} \subset A$ and $B^{\prime} \subset B$ with $\mu\left(A \backslash A^{\prime}\right)=\mu\left(B \backslash B^{\prime}\right)=0$ and a homeomorphism $S: A^{\prime} \rightarrow B^{\prime}$ such that $S \in[[T]]$ top.

Proof. The case (i) was demonstrated in [dJS].

We now consider the case (ii). We construct $S$ via an inductive process. Since $T$ is of type $I I I$, there are $N>0$ and mutually disjoint Borel subsets $\left(A_{i}\right)_{|i|<N}$ of $A$ such that $0.5 \mu(A)<\sum_{|i|<N} \mu\left(A_{i}\right)<\mu(A)$, the sets $B_{i}:=T^{i} A_{i},|i|<N$, are mutually disjoint Borel subsets of $B$ and $0.5 \mu(B)<\sum_{|i|<N} \mu\left(B_{i}\right)<\mu(B)$ [Kr2], [HO]. Fix a small $\epsilon>0$ and find mutually disjoint clopen subsets $A_{i}^{0} \subset A$ such that $\mu\left(A_{i} \triangle A_{i}^{0}\right)<\epsilon \mu\left(A_{i}\right)$, the subsets $B_{i}^{0}:=T^{i} A_{i}^{0},|i|<N$, are mutually disjoint (clopen) subsets of $B$ and $\mu\left(B_{i} \triangle B_{i}^{0}\right)<\epsilon \mu\left(B_{i}\right)$. Now we can define a homeomorphism - a 'piece' of $S$ - from the clopen subset $A^{0}:=\bigsqcup_{|i|<N} A_{i}^{0}$ of $A$ 
onto the clopen subset $B^{0}:=\bigsqcup_{|i|<N} B_{i}^{0}$ of $B$ by setting $S x=T^{i} x$ if $x \in A_{i}^{0}$. Notice that $\mu\left(A^{0}\right)>0.5 \mu(A)$ and $\mu\left(B^{0}\right)>0.5 \mu(B)$ if $\epsilon$ is sufficiently small. This completes the first step of the inductive procedure. Now repeat the same for the pair of clopen subsets $A \backslash A^{0}$ and $B \backslash B^{0}$ and so on. It remains to concatenate the 'pieces' of $S$ that were defined in all steps to obtain a homeomorphism $\phi \in[[T]]_{\text {top }}$ from an open subset $A^{\prime}$ of $A, \mu\left(A^{\prime}\right)=\mu(A)$, onto an open subset $B^{\prime}$ of $B, \mu\left(B^{\prime}\right)=\mu(B)$. The graph of $S$ is a subset of $\mathcal{R}_{T}$ because each 'piece' of $S$ enjoys this property.

Corollary 1.5. If $T$ is of type III and $A$ is virtually clopen then $T_{A}$ is almost continuously orbit equivalent to $T$.

For some non-clopen subsets $A \subset X$, the induced system $T_{A}$ can be well defined too. However, it may not be almost continuously orbit equivalent to $T$. We illustrate this by the following example.

Example 1.6. Let $C_{n}:=\{0,1\}$ if $n$ is odd and $C_{n}:=\left\{0,1, \ldots, n^{2}+1\right\}$ if $n$ is even. Let $T$ stand for the odometer on the infinite product space $X=\prod_{n=1}^{\infty} C_{n}$ endowed with the infinite product topology $\left(C_{n}\right.$ are all endowed with the discrete topology). Then $T$ is a homeomorphism of a 0 -dimensional compact metric space (Cantor set). Fix two reals $0<\lambda, \alpha<1$. We define a probability measure $\mu_{n}$ on $C_{n}$ by setting $\mu_{n}(0)=(1+\lambda)^{-1}$ and $\mu_{n}(1)=\lambda /(1+\lambda)$ for odd $n$ and for even $n$

$$
\mu_{n}(i)= \begin{cases}1 /\left(n^{2}(1+\alpha)\right), & \text { if } i=0 \\ \alpha /\left(n^{2}(1+\alpha)\right), & \text { if } i=1 \\ n^{-2}, & \text { if } 1<i \leq n^{2}+1 .\end{cases}
$$

It is easy to see that the product measure $\mu=\bigotimes_{n=1}^{\infty} \mu_{n}$ on $X$ is non-atomic. Moreover, $T$ is $\mu$-non-singular and ergodic. Let $A:=C_{1} \times C_{2}^{\prime} \times C_{3} \times C_{4}^{\prime} \times \ldots$, where $C_{n}^{\prime}:=\left\{2,3, \ldots, n^{2}+1\right\}$. Then $A$ is a compact meager subset of $X$ and $\mu(A)>0$. It is easy to see that $T_{A}$ is a well defined homeomorphism of $A$, in fact an odometer, and the dynamical system $\left(T_{A}, \mu \uparrow A\right)$ is of type $I I I_{\lambda}$. Hence $T$ is also of type $I I I_{\lambda}$. Notice that the map $x \mapsto \rho_{\mu}(x, T x)$ is continuous everywhere on $X$ except of a singleton. It is easy to verify that $\{\log \lambda, \log \alpha\} \subset r_{\text {top }}\left(\rho_{\mu}\right)$. Since $\rho_{\mu}$ takes values in the group generated by $\log \lambda$ and $\log \alpha$, it follows that $r_{\text {top }}\left(\rho_{\mu}\right)$ is the closure of this group. Thus $r_{\text {top }}\left(\rho_{\mu}\right) \neq r\left(\rho_{\mu}\right)$ whenever $\alpha$ is not a power of $\lambda$. It follows that $T$ and $T_{A}$ while being orbit equivalent are not almost continuously orbit equivalent.

Lemma 1.7. If $r_{\text {top }}\left(\rho_{\mu}\right) \cap[a, b]=\emptyset$ then there is a clopen subset $A \subset X$ with $\rho_{\mu}(x, y) \notin[a, b]$ for all $(x, y) \in \mathcal{R}_{T} \cap(A \times A)$.

Proof. We first find for each $t \in[a, b]$, a clopen subset $B_{t} \subset X$ and $\epsilon_{t}>0$ such that $\rho_{\mu}(x, y) \notin\left(t-\epsilon_{t}, t+\epsilon_{t}\right)$ for all $\mathcal{R}_{T} \cap\left(B_{t} \times B_{t}\right)$. Select a finite collection of the intervals $\left(t-\epsilon_{t} / 2, t+\epsilon_{t} / 2\right)$ that cover the entire $[a, b]$ and enumerate them as $\left(t_{i}-\epsilon_{i} / 2, t_{i}+\epsilon_{i} / 2\right), 1 \leq i \leq l$, with corresponding sets $B_{i}$. Since $T$ is ergodic and the Radon-Nikodym derivative $d \mu \circ T / d \mu$ is continuous, there exist a clopen subset $C_{2} \subset B_{1}$ and $k \in \mathbb{Z}$ with $T^{k} C_{2} \subset B_{2}$ and the map $C_{2} \ni x \mapsto \rho_{\mu}\left(x, T^{k} x\right)$ is close to a constant. Take $(x, y) \in \mathcal{R}_{T} \cap\left(C_{2} \times C_{2}\right)$. Then

$$
\begin{aligned}
& \rho_{\mu}(x, y) \notin\left(t_{1}-\epsilon_{1}, t_{1}+\epsilon_{1}\right) \text { and } \\
& \rho_{\mu}(x, y)=\rho_{\mu}\left(x, T^{k} x\right)+\rho_{\mu}\left(T^{k} x, T^{k} y\right)-\rho_{\mu}\left(y, T^{k} y\right) \notin\left(t_{2}-\epsilon_{2}, t_{2}+\epsilon_{2}\right) .
\end{aligned}
$$


Continue in this fashion to get clopen sets $C_{2} \supset C_{3} \supset \cdots \supset C_{l}$ such that for some $k_{i}$ we have $T^{k_{i}} C_{i} \subset B_{i}$ and the map $C_{i} \ni x \mapsto \rho_{\mu}\left(x, T^{k_{i}} x\right)$ is close to a constant. Setting $A:=C_{l}$ proves the lemma.

Lemma 1.8. There is an inessential reduction $\widetilde{X} \subset X$ and a countable base $\left(O_{i}\right)_{i=1}^{\infty}$ of the topology in $\mathbb{R}$ such that the sets

$$
\left\{x \in \widetilde{X} \mid \rho_{\mu}(x, T x) \in O_{i}\right\}, \quad i=1,2, \ldots
$$

are clopen in $\widetilde{X}$ (in the relative topology).

Proof. We first notice that there are no more than countably many $t \in \mathbb{R}$, say bad $t$, such that the closed subsets

$$
F_{t}:=\{x \in \widetilde{X} \mid \rho(x, T x)=t\}
$$

have positive measure. Chose a countable family of intervals $\left(O_{i}\right)_{i=1}^{\infty}$ in $\mathbb{R}$ which generate the topology and whose end points are not bad. Let $F$ be the smallest $T$-invariant subset that contains $F_{t}$ when $t$ runs through the end points of all $O_{i}$, $i=1,2, \ldots$. It remains to put $\widetilde{X}:=X \backslash F$.

Definition 1.9. Given $r>0$, we let $I_{r}:=\{0,1, \ldots, r-1\}$ and endow it with the discrete topology. A subrelation $\mathcal{S} \subset \mathcal{R}_{T}$ is called $r$-uniform if there are a clopen subset $B \subset X$ and a homeomorphism $h: I_{r} \times B \rightarrow X$ such that $h\left(I_{r} \times\{b\}\right)=\mathcal{S}(b)$ and $h(0, b)=b$ for $b \in B$ and for each $i$ the map

$$
B \ni h(0, b) \mapsto h(i, b) \in h(\{i\} \times B)
$$

belongs to $[[T]]_{\text {top. }}$ We call $B$ an $\mathcal{S}$-fundamental subset and $h$ an $(\mathcal{S}, B)$-splitting homeomorphism. Associated to $\mathcal{S}$ we have a finite subgroup $G_{\mathcal{S}}$ of $[T]_{\text {top }}$ corresponding to the action of the permutation group $S_{r}$ on $I_{r}$. We also have a natural projection $p: X \rightarrow B$ mapping $h(i, b)$ to $h(0, b)$ for each $i$.

It is worth noting that neither $B$ nor $h$ is determined uniquely by $\mathcal{S}$ or $(\mathcal{S}, B)$ respectively. In a similar way, $G_{\mathcal{S}}$ depends really on $(\mathcal{S}, B, h)$ and not only on $\mathcal{S}$ alone. Each $q$-uniform subrelation $\mathcal{T}$ of $\mathcal{R}_{T} \cap(B \times B)$ extends canonically, via $\mathcal{S}$, to an $r q$-uniform subrelation

$$
\widetilde{\mathcal{T}}:=\left\{(x, y) \in \mathcal{R}_{T} \mid(p(x), p(y)) \in \mathcal{T}\right\}
$$

of $\mathcal{R}_{T}$. We call it the natural extension of $\mathcal{T}$ to $X$. Each $\mathcal{T}$-fundamental subset is also a $\widetilde{\mathcal{T}}$-fundamental subset.

The following topological version of the non-singular Rokhlin lemma can be proved in the same way as its measure-preserving counterpart (Lemma 6, [dJS]).

Lemma 1.10. Given $\epsilon>0$ and an integer $N>0$, there is a clopen subset $A \subset X$ such that the subsets $T^{i} A, i=0, \ldots, N$, are mutually disjoint and $\mu(X \backslash$ $\left.\bigsqcup_{i=0}^{N} T^{i} A\right)<\epsilon$.

We need this lemma to obtain the following result. 
Lemma 1.11. Let $\mathcal{S}$ be a uniform subrelation of $\mathcal{R}_{T}$ and let $B \subset X$ be an $\mathcal{S}$ fundamental subset. For each $\epsilon>0$, there exists a uniform subrelation $\mathcal{T} \subset \mathcal{R}_{T} \cap$ $(B \times B)$ such that $\mu(\{x \in X \mid T x \in \widetilde{\mathcal{T}}(x)\})>1-2 \epsilon$.

Proof. Take $\delta>0$ such that whenever $C$ is a subset of $B$ with $\mu(C)<\delta \mu(B)$ then $\mu\left(\bigcup_{x \in C} \mathcal{S}(x)\right)<\epsilon$.

Denote by $T_{B}: B \rightarrow B$ the induced homeomorphism. Select a clopen subset $A \subset B$ and a positive integer $M$ such that $\mu(A)>(1-\delta / 2) \mu(B)$ and $T \mathcal{S}(x) \subset$ $\bigsqcup_{|i|<M} \mathcal{S}\left(T_{B}^{i} x\right)$ for each $x \in A$. We deduce from the Lemma 1.10 that for a sufficiently large $N>M$, there exists a clopen subset $B_{0} \subset B$ such that the subsets $T_{B}^{i} B_{0}, i=0, \ldots, N-1$ are mutually disjoint and

$$
\mu\left(B \backslash \bigsqcup_{i=M}^{N-M} T_{B}^{i} B_{0}\right)<\delta \mu(B) / 2 .
$$

Partition the subset $B \backslash \bigsqcup_{i=0}^{N-1} T_{B}^{i} B_{0}$ into $N$ non-empty clopen subsets $D_{j}, 0 \leq$ $j<N$. By Lemma 1.4(ii), there are homeomorphisms $\gamma_{j}: D_{0} \rightarrow D_{j}$ belonging to $[[T]]_{\text {top }}$. Now we define an equivalence relation $\mathcal{T}$ on $B$ by setting $(x, y) \in \mathcal{T}$ if either

$-x=T_{B}^{i} z$ and $y=T_{B}^{j} z$ for some $z \in B_{0}$ and $0 \leq i, j<N$ or
$-x=\gamma_{i} z$ and $y=\gamma_{j} z$ for some $z \in D_{0}$ and $0 \leq i, j<N$.

It is obvious that $\mathcal{T}$ is an $N$-uniform subrelation of $\mathcal{R}_{T} \cap(B \times B)$. Let $E$ stand for the union of all subsets $\mathcal{S}(x)$ when $x$ runs through $A \cap \bigsqcup_{M<i<N-M} T_{B}^{i} B_{0}$. Then $\mu(E)>1-\epsilon$ and $T x \in \widetilde{\mathcal{T}}(x)$ for all $x \in E$.

We remark that the set $O:=\{x \in X \mid T x \in \widetilde{\mathcal{T}}(x)\}$ in the conclusion of Lemma 1.11 is automatically (virtually) clopen and that $\left.T\right|_{O} \in\left[\left[G_{\widetilde{\mathcal{T}}(x)}\right]\right]_{\text {top }}$.

\section{TYPe $I I I_{\lambda}$ WITH $0<\lambda<1$}

In this section we prove Theorem 0.1 in the case when $T$ is of type $I I I_{\lambda}$ with $0<\lambda<1$.

Proposition 2.1. Let $(X, \tau, \mu, T)$ be a dynamical system with $r_{\mathrm{top}}\left(\rho_{\mu}\right)=\log \lambda$. $\mathbb{Z}$. (We do not assume that $\left.r\left(\rho_{\mu}\right)=\log \lambda \cdot \mathbb{Z}\right)$. Then there exists an equivalent probability measure $\widetilde{\mu}$ such that $\rho_{\widetilde{\mu}}(x, y) \in \log \lambda \cdot \mathbb{Z}$ for all $(x, y) \in \mathcal{R}_{T}$ and $d \widetilde{\mu} / d \mu$ is continuous (when restricted to an inessential reduction).

Proof. We first note that if there are $\eta>0$ and a clopen subset $A \subset X$ such that

$$
\rho_{\mu}(x, y) \notin[\log \lambda+\eta,-\eta] \text { for all }(x, y) \in \mathcal{R}_{T} \cap(A \times A)
$$

then $\rho_{\mu}(x, y) \in \log \lambda \cdot \mathbb{Z}+(-\eta, \eta)$ for all $(x, y) \in \mathcal{R}_{T} \cap(A \times A)$.

Fix a summable sequence $\left(\eta_{i}\right)_{i=0}^{\infty}$ of positive reals. It follows from Lemma 1.7 and the last remark that there exists a clopen subset $A_{0} \subset X$ such that

$$
\rho_{\mu}(x, y) \in \log \lambda \cdot \mathbb{Z}+\left(-\eta_{1}, \eta_{1}\right) \text { for all }(x, y) \in \mathcal{R}_{T} \cap\left(A_{0} \times A_{0}\right) .
$$

Passing to an inessential reduction, we can represent $X$ as $X=\bigsqcup_{k=0}^{\infty} A_{k}$, where $A_{k}$ is clopen and $T^{-k} A_{k} \subset A_{0}$. We now apply Lemma 1.8 to find a continuous function $\phi_{1}: X \rightarrow(\log \lambda,-\log \lambda)$ such that $\phi_{1}=0$ on $A_{0}^{(i)}$ and

$$
\phi_{1}(x)+\rho_{\mu}\left(x, T^{-k} x\right) \in \log \lambda \cdot \mathbb{Z}+\left(-\eta_{1}, \eta_{1}\right) \text { for all } x \in A_{k} .
$$


Let $\mu_{1}:=\exp \left(\phi_{1}\right) \cdot \mu$. Then it is easy to verify that

$$
\rho_{\mu_{1}}(x, y) \in \log \lambda \cdot \mathbb{Z}+\left(-3 \eta_{1}, 3 \eta_{1}\right) \text { for all }(x, y) \in \mathcal{R}_{T} .
$$

We now repeat the process with $\mu_{1}$ in place of $\mu$ to obtain $\mu_{2}=\exp \left(\phi_{2}\right) \mu_{1}$ such that

$$
\rho_{\mu_{2}}(x, y) \in \log \lambda \cdot \mathbb{Z}+\left(-3 \eta_{2}, 3 \eta_{2}\right) \text { for all }(x, y) \in \mathcal{R}_{T} .
$$

However, now because of (2-1) we see that $\phi_{2}$ may be chosen with values in $\left(-3 \eta_{1}, 3 \eta_{1}\right)$. Continuing in this fashion we obtain a sequence $\left(\mu_{i}\right)_{i=1}^{\infty}$ of measures such that $\mu_{i+1}=\exp \left(\phi_{i}\right) \mu_{i}, \phi_{i}$ is continuous, $\left|\phi_{i+1}\right|<3 \eta_{i}$ and

$$
\rho_{\mu_{i}}(x, y) \in \log \lambda \cdot \mathbb{Z}+\left(-3 \eta_{i}, 3 \eta_{i}\right) \text { for all }(x, y) \in \mathcal{R}_{T} .
$$

The series $\sum_{i>0} \phi_{i}$ converges uniformly to a continuous function $\phi$ and we have only to set $\widetilde{\mu}=\exp (\phi) \mu$ to complete the proof. We note that $\widetilde{\mu}$ (as well as $\widetilde{\mu}_{i}$ ) can be infinite by construction. Nevertheless it is locally finite. Hence, after multiplying by a suitable continuous function taking values in $\{n \log \lambda: n \in \mathbb{Z}\}$, we may assume without loss of generality that $\widetilde{\mu}$ is finite.

We will call $\widetilde{\mu}$ a special measure for $T$.

Remark 2.2. If $r_{\text {top }}\left(\rho_{\mu}\right)=\log \lambda \cdot \mathbb{Z}$ and $\widetilde{\mu}$ is special then the "measure-preserving" subrelation $\mathcal{S}:=\left\{(x, y) \in \mathcal{R}_{T} \mid \rho_{\widetilde{\mu}}(x, y)=0\right\}$ of $\mathcal{R}_{T}$ is ergodic if and only if $r\left(\rho_{\mu}\right)=\log \lambda \cdot \mathbb{Z}$.

To see this let us agree to say that measurable subsets $A$ and $B$ communicate via $l \in \mathbb{Z}$ if there are subsets $A^{\prime} \subset A, B^{\prime} \subset B$ of positive measure and $k \in \mathbb{Z}$ such that $T^{k} A^{\prime}=B^{\prime}$ and $\rho_{\widetilde{\mu}}\left(x, T^{k} x\right)=l \log \lambda$ for all $x \in A^{\prime}$.

Now if $r\left(\rho_{\mu}\right)=\log \lambda \cdot \mathbb{Z}$ and $A$ and $B$ are measurable sets of positive measure then certainly $A$ and $B$ communicate via some $l$. Since $T$ is type $I I I_{\lambda}$ and $\widetilde{\mu}$ is special we also have that every subset of $B$ of positive measure communicates with itself via $-l$ and it follows that $A$ and $B$ communicate via 0 , as desired.

On the other hand if $\mathcal{S}$ is ergodic, since $r_{\text {top }}\left(\rho_{\mu}\right)=\log \lambda \cdot \mathbb{Z}=r_{\text {top }}\left(\rho_{\widetilde{\mu}}\right)$ then there certainly exist (clopen) $A$ and $B$ which communicate via 1 . If $C$ is an arbitrary measurable set of positive measure then by ergodicity of $\mathcal{S}$ each subset of $C$ of positive measure communicates with both $A$ and $B$ via 0 and 'by composition' it follows that $C$ communicates with $C$ via 1 , showing that $\lambda \in r\left(\rho_{\widetilde{\mu}}\right)=r\left(\rho_{\mu}\right)$. Thus $r\left(\rho_{\mu}\right) \supset \log \lambda \cdot \mathbb{Z}$ and we already have the opposite inclusion.

The following statement is a refinement of Lemma 1.4(ii) for type $I I I_{\lambda}$ systems.

Lemma 2.3. Let $T$ be of type $I I I_{\lambda}, r_{\text {top }}\left(\rho_{\mu}\right)=\log \lambda \cdot \mathbb{Z}$ and $\mu$ a special measure. Given two clopen subsets $A, B \subset X$ with $\mu(B)=\lambda^{k} \mu(A)$, there exists a homeomorphism $S: A \rightarrow B$ such that $S \in[[T]]_{\text {top }}$ and $\rho_{\mu}(x, S x)=k \log \lambda$ for all $x \in A$.

Proof. Let $n(x):=\min \left\{n>0 \mid \rho_{\mu}\left(x, T^{n} x\right)=0\right\}$ and $R x:=T^{n(x)} x$. Since $\rho_{\mu}$ is continuous and takes values in the discrete set $\log \lambda \cdot \mathbb{Z}$, it is easy to see that $n$ is virtually continuous so that $R \in[[T]]_{\text {top }}$. The $R$-orbit equivalence relation co-incides with $\mathcal{S}$, the measure-preserving subrelation. Hence $R$ is ergodic by Remark 2.2 . Since $T$ is ergodic of type $I I I_{\lambda}$, there are a non-empty clopen subset $A_{0} \subset X$ and $l \in \mathbb{Z}$ such that $\rho_{\mu}\left(x, T^{l} x\right)=k \log \lambda$ for all $x \in A_{0}$. In view of Lemma 1.2 we may assume that $\mu\left(A_{0}\right)=N^{-1} \mu(A)$ for some $N \in \mathbb{Z}$. Again by Lemma 1.2, there are 
partitions $A=\bigsqcup_{i=1}^{N} A_{i}$ and $B=\bigsqcup_{i=1}^{N} B_{i}$ of $A$ and $B$ into clopen subsets $A_{i}$ and $B_{i}$ such that $\mu\left(A_{i}\right)=N^{-1} \mu\left(A_{0}\right)$ and $\mu\left(B_{i}\right)=N^{-1} \mu\left(B_{0}\right)$ for all $i$. By Lemma 1.4(i), there exist homeomorphisms $\gamma_{i}: A_{i} \rightarrow A_{0}$ and $\delta_{i}: B_{i} \rightarrow T^{l} A_{0}$ with $\gamma_{i}, \delta_{i} \in[[R]]_{\text {top }}$. It remains to set $S x:=\delta_{i}^{-1} T^{l} \gamma_{i} x$ for all $x \in A_{i}, i=1, \ldots, N$.

Lemma 2.4. Let $(\mu, T)$ be as in Lemma 2.3. Let $D, \widetilde{D}$ be two countable discrete spaces and $B$ a clopen subset of $X$. Let $\nu$ be a finite measure on $I_{r} \times \widetilde{D}$ such that $\nu(i, d) / \nu(j, d)$ is a power of $\lambda$ for all $i, j \in I_{r}$ and $d \in \widetilde{D}$. Given continuous onto maps $u: B \rightarrow D$ and $\widetilde{v}: I_{r} \times \widetilde{D} \rightarrow D$ such that $\mu \circ u^{-1}=\nu \circ \widetilde{v}^{-1}$, there are an $r$-uniform equivalence relation $\mathcal{S} \subset \mathcal{R}_{T}$ on $B$, an $\mathcal{S}$-fundamental subset $\widetilde{B} \subset B$, an $(\mathcal{S}, \widetilde{B})$-splitting homeomorphism $v: I_{r} \times \widetilde{B} \rightarrow B$ and a measure-preserving continuous onto map $\widetilde{u}: \widetilde{B} \rightarrow \widetilde{D}$ such that the following diagram commutes

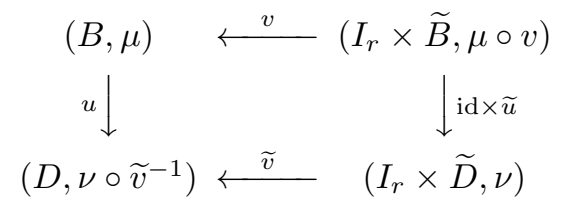

and $\rho_{\mu}(v(j, \widetilde{b}), v(i, \widetilde{b}))=\log (\nu(j, \widetilde{u}(\widetilde{b})) / \nu(i, \widetilde{u}(\widetilde{b})))$ for all $i, j \in I_{r}$ and $\widetilde{b} \in \widetilde{B}$. Here $\widetilde{B}$ and $\widetilde{D}$ are identified with the 'bottom' levels $\{0\} \times \widetilde{B}$ and $\{0\} \times \widetilde{D}$ of $I_{r} \times \widetilde{B}$ and $I_{r} \times \widetilde{D}$ respectively equipped with the induced measures.

Proof. Since $\mu\left(u^{-1}(d)\right)=\nu\left(\widetilde{v}^{-1}(d)\right)$ for each $d \in D$, it follows from Lemma 1.2 that there exists a partition $B=\bigsqcup_{(i, \widetilde{d}) \in I_{r} \times \widetilde{D}} B_{i, \widetilde{d}}$ of $B$ into clopen subsets such that $\mu\left(B_{i, \widetilde{d}}\right)=\nu(i, \widetilde{d})$ and $u\left(B_{i, \widetilde{d}}\right)=\widetilde{v}(i, \widetilde{d})$ for all $(i, \widetilde{d}) \in I_{r} \times \widetilde{D}$. Hence the ratio $\mu\left(B_{i, \widetilde{d}}\right) / \mu\left(B_{0, \widetilde{d}}\right)$ is a power of $\lambda$ and then Lemma 2.3 yields that there exists a homeomorphism $\gamma_{i, \widetilde{d}}: B_{i, \widetilde{d}} \rightarrow B_{0, \widetilde{d}}$ such that $\gamma_{i, \widetilde{d}} \in[[T]]_{\text {top }}$ and

$$
\rho_{\mu}\left(x, \gamma_{i, \widetilde{d}} x\right)=\log \left(\mu\left(B_{0, \widetilde{d}}\right) / \mu\left(B_{i, \widetilde{d}}\right)\right) \text { for all } x \in B_{i, \widetilde{d}} .
$$

Let $\mathcal{S}$ be the equivalence relation generated by the graphs of all $\gamma_{i, \widetilde{d}}, i \in I_{r}, \widetilde{d} \in \widetilde{D}$. It is $r$-uniform and the subset $\widetilde{B}:=\bigsqcup_{d \in \widetilde{D}} B_{0, \widetilde{d}} \subset B$ is $\mathcal{S}$-fundamental. It remains to put $\widetilde{u}=\widetilde{d}$ on $B_{0, \widetilde{d}}$ and $v(i, b)=\gamma_{i, \widetilde{d}}^{-1} b$ if $(i, b) \in I_{r} \times B_{0, \widetilde{d}}$.

Now we state and prove the main result of this section - part (i) of Theorem 0.1.

Theorem 2.5. Let $(X, \tau, \mu, T)$ and $\left(X^{\prime}, \tau^{\prime}, \mu^{\prime}, T^{\prime}\right)$ be two dynamical systems of type $I I I_{\lambda}, 0<\lambda<1$, and $r_{\mathrm{top}}\left(\rho_{\mu}\right)=r_{\mathrm{top}}\left(\rho_{\mu^{\prime}}\right)=\log \lambda \cdot \mathbb{Z}$. Then these systems are almost continuously orbit equivalent.

Proof. By Proposition 2.1 we may assume without loss of generality that $\mu$ and $\mu^{\prime}$ are special for $T$ and $T^{\prime}$ respectively. Let $m$ and $m^{\prime}$ stand for the complete metrics compatible with the topologies on $X$ and $X^{\prime}$ respectively. Fix a sequence $\epsilon_{n} \rightarrow 0$. Suppose that we have inessential reductions $X_{0} \subset X$ and $X_{0}^{\prime} \subset X^{\prime}$, a sequence of positive integers $r_{n}$ and two nested sequences of clopen subsets

$$
X_{0}=: B_{0} \supset B_{1} \supset \cdots, \quad X_{0}^{\prime}=: B_{0}^{\prime} \supset B_{1}^{\prime} \supset \cdots
$$

in $X_{0}$ and $X_{0}^{\prime}$ respectively such that $B_{n}$ is a fundamental subset of an $r_{n}$-uniform equivalence relation $\mathcal{R}_{n} \subset \mathcal{R}_{T} \cap\left(B_{n-1} \times B_{n-1}\right)$ and $B_{n}^{\prime}$ is a fundamental subset of 
an $r_{n}$-uniform equivalence relation $\mathcal{R}_{n}^{\prime} \subset \mathcal{R}_{T^{\prime}} \cap\left(B_{n-1}^{\prime} \times B_{n-1}^{\prime}\right)$. Suppose also that we can choose for any $n$,

- an $\left(\mathcal{R}_{n}, B_{n}\right)$-splitting homeomorphism $h_{n}: I_{r_{n}} \times B_{n} \rightarrow B_{n-1}$,

- a measure preserving continuous map $p_{n}$ of $B_{n}$ onto a discrete countable set $D_{n}$,

- a measure preserving onto map $g_{n}: I_{r_{n}} \times D_{n} \rightarrow D_{n-1}$,

- similar objects $h_{n}^{\prime}, p_{n}^{\prime}, g_{n}^{\prime}$ for the second system

in such a way that the infinite diagram

$$
\begin{aligned}
& X_{0} \stackrel{h_{1}}{\longleftarrow} I_{r_{1}} \times B_{1} \stackrel{\mathrm{id} \times h_{2}}{\longleftarrow} I_{r_{1}} \times I_{r_{2}} \times B_{2} \stackrel{\mathrm{id} \times \mathrm{id} \times h_{3}}{\longleftarrow} \ldots \\
& \operatorname{id} \times p_{1} \downarrow \quad \operatorname{id} \times \operatorname{id} \times p_{2} \downarrow \\
& I_{r_{1}} \times D_{1} \stackrel{\mathrm{id} \times g_{2}}{\longleftarrow} I_{r_{1}} \times I_{r_{2}} \times D_{2} \stackrel{\mathrm{id} \times \mathrm{id} \times g_{3}}{\longleftarrow} \cdots \\
& \operatorname{id} \times p_{1}^{\prime} \uparrow \quad \operatorname{id} \times \operatorname{id} \times p_{2}^{\prime} \uparrow \\
& X_{0}^{\prime} \stackrel{h_{1}^{\prime}}{\longleftarrow} I_{r_{1}} \times B_{1}^{\prime} \stackrel{\operatorname{id} \times h_{2}^{\prime}}{\longleftarrow} I_{r_{1}} \times I_{r_{2}} \times B_{2}^{\prime} \stackrel{\operatorname{id} \times \mathrm{id} \times h_{3}^{\prime}}{\longleftarrow} \cdots
\end{aligned}
$$

commutes.

Since each arrow in the upper line is a homeomorphism the measure $\mu$ in $X_{0}$ induces a measure on each space in the upper line and these measures in turn project onto the spaces in the middle line. We also require of (2-2) that these measures on the middle line co-incide with the measures coming in the same way from the lower line. Thus every space in the picture carries a measure and the arrows are all measure-preserving. For any $n$, we have a continuous onto map from $X_{0}$ to $I_{r_{1}} \times \cdots \times I_{r_{n}} \times D_{n}$ which is a composition of $n$ reversed horizontal arrows plus a vertical arrow. We denote this map by $\pi_{n}$ and view it as a partition of $X_{0}$ into clopen subsets. We will denote by $\widetilde{\mathcal{R}}_{n}$ the natural extension of $\mathcal{R}_{n}$ to $X_{0}$.

We further require of (2-2) that

$(\triangle)$ for each odd $n$, the diameter of (every atom of) $\pi_{n}$ is less than $\epsilon_{n}$ with respect to $m$;

$(\diamond)$ for each odd $n$ there is a clopen subset $O_{n} \subset X_{0}$ such that $\mu\left(O_{n}\right)>1-\epsilon_{n}$ and $T x \in \widetilde{\mathcal{R}}_{n}(x)$ for all $x \in O_{n}$.

$(\nabla)$ for all $n$ the Radon-Nikodym derivative on $\mathcal{R}_{n}$ is equivariant under $p_{n}$, i.e. given $d \in D_{n}$ and $i, j \in I_{r_{n}}$, the map $p_{n}^{-1}(d) \ni b \mapsto \rho_{\mu}\left(h_{n}(i, b), h_{n}(j, b)\right)$ is constant.

(D) similar conditions hold for the 'bottom line' of (2-1) but with "odd" replaced by "even" $n$ in $(\triangle)$ and $(\diamond)$.

Under all these assumptions on (2-2) we proceed to construct our orbit equivalence. Denote by $Z$ the topological and measure-theoretic inverse limit of the middle line in (2-2) and denote by $\nu$ the measure on $Z$. It is obvious that $Z$ is a 0 -dimensional Polish space. An element $z$ of $Z$ is a sequence $\left(z_{n}\right)_{n=1}^{\infty}$ such that $z_{n}=\left(i_{1}, \ldots, i_{n}, d_{n}\right)$ for some $i_{k} \in I_{r_{k}}, 1 \leq k \leq n$, and $d_{n} \in D_{n}$ with $d_{n}=g_{n+1}\left(i_{n+1}, d_{n+1}\right), n \geq 1$. Since (2-2) commutes and all the arrows are onto, there is a continuous map $\phi: X_{0} \rightarrow Z$ whose image is dense in $Z$. It follows from $(\triangle)$ that $\phi$ is one-to-one and open as a map from $X_{0}$ to $\phi\left(X_{0}\right)$ endowed with the induced topology, that is $\phi$ is a homeomorphism onto its image. Since $X_{0}$ is Polish, $\phi\left(X_{0}\right)$ is Polish. It follows by the Alexandrov-Hopf theorem that $\phi\left(X_{0}\right)$ is a $G_{\delta}$ in $Z$. Note also that $\mu \circ \phi^{-1}=\nu$. 
We let $G$ denote the countable subgroup of $[T]_{\text {top }}$ which is the increasing union of the finite subgroups $G_{n}:=G_{\widetilde{\mathcal{R}}_{n}}$. We claim that, because of $(\diamond), T \in[G]_{\text {top }}$, so that in fact $[T]_{\text {top }}=[G]_{\text {top }}$. To see this note that for $n$ odd we have $\left.T\right|_{O_{n}} \in\left[\left[G_{n}\right]\right]_{\text {top }}$, that is $T x=g_{n}(x)(x)$ where $g_{n}: O_{n} \rightarrow G_{n}$ is virtually continuous. (See the remark after the proof of Lemma 1.11.) Let $O=\bigcup_{n \geq 0} O_{2 n+1}$, a virtually open set of full measure. If we define $g: O \rightarrow G$ by $g(x)=g_{2 n+1}(x)$ for $x \in O_{2 n+1} \backslash O_{2 n-1}$ then $g$ is virtually continuous, since each $O_{2 n+1} \backslash O_{2 n-1}$ is clopen and $T x=g(x) x$ for $x \in O$. This shows that $T \in[G]_{\text {top }}$ as claimed.

The permutation group $S_{r_{1} r_{2} \ldots r_{n}}$ acts on $I_{r_{1}} \times \cdots \times I_{r_{n}}$ and these permutations lift to homeomorphisms of $Z$, comprising a group which we denote $H_{n}$. It is clear that $\phi G_{n} \phi^{-1}=H_{n}$ so $\phi G \phi^{-1}=H$ where $H=\bigcup_{n} H_{n}$. Thus we have

$$
\phi[T]_{\mathrm{top}} \phi^{-1}=\phi[G]_{\mathrm{top}} \phi^{-1}=[H]_{\mathrm{top}} .
$$

In a similar way — using $(\mathcal{Q})$ —one can define a measure-preserving map $\phi^{\prime}: X_{0}^{\prime} \rightarrow$ $Z$ which is a homeomorphism onto a dense $G_{\delta}$ such that

$$
\phi^{\prime}[T]_{\mathrm{top}}\left(\phi^{\prime}\right)^{-1}=[H]_{\mathrm{top}}
$$

and $\mu^{\prime} \circ\left(\phi^{\prime}\right)^{-1}=\nu$. Now the map $\psi=\left(\phi^{\prime}\right)^{-1} \circ \phi$ is virtually everywhere defined and $\psi[T]_{\text {top }} \psi^{-1}=[S]_{\text {top }}$ so $\psi$ is a (measure-preserving) almost continuous orbit equivalence.

It remains to explain how to construct $(2-2)$ satisfying $(\triangle),(\diamond),(\nabla)$ and $(\nabla)$. This will be done via an inductive process. Suppose we have already constructed (2-2) right up to an odd index $n$. Moreover, suppose that the 'bottom square' of (2-2) with index $n+1$ also has been constructed. Thus the following fragment of (2-2) commutes:

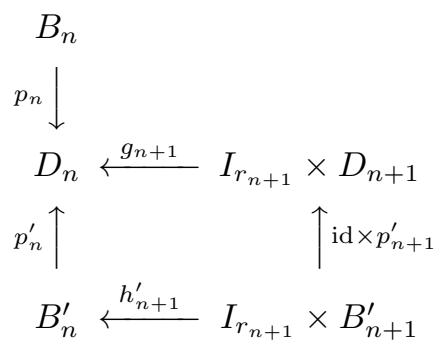

Recall that the arrows are assumed measure preserving. Since $\mu^{\prime}$ is special and $(\nabla)$ and $(\nabla)$ hold, it follows that the measure on $I_{r_{n+1}} \times D_{n+1}$, say $\nu_{n+1}$, is such that $\nu_{n+1}(i, d) / \nu_{n+1}(j, d)$ is a power of $\lambda$ for all $i, j \in I_{r_{n+1}}$ and $d \in D_{n+1}$. Then we can apply Lemma 2.4 to find an $r_{n+1}$-uniform subrelation $\mathcal{R}_{n+1} \subset \mathcal{R}_{T} \cap\left(B_{n} \times B_{n}\right)$, an $\mathcal{R}_{n+1}$-fundamental subset $B_{n+1} \subset B_{n}$, a $\left(\mathcal{R}_{n+1}, B_{n+1}\right)$-splitting homeomorphism $h_{n+1}: I_{r_{n+1}} \times B_{n+1} \rightarrow B_{n}$ and a measure preserving continuous onto map $p_{n+1}$ : $B_{n+1} \rightarrow D_{n+1}$ such that the diagram

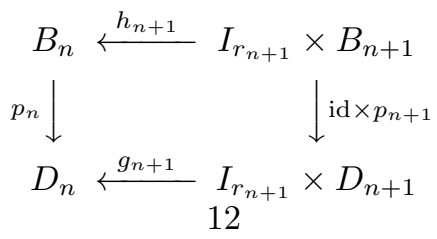


commutes and the Radon-Nikodym derivative on $\mathcal{R}_{n+1}$ is equivariant under $p_{n+1}$ (i.e. $(\nabla)$ holds for $n+1)$. Now apply Lemma 1.11 to find $r_{n+2}>0$, an $r_{n+2}$-uniform subrelation $\mathcal{R}_{n+2} \subset \mathcal{R}_{T} \cap\left(B_{n+1} \times B_{n+1}\right)$ and a subset $O_{n+2} \subset X_{0}$ satisfying $(\diamond)$. Select an $\mathcal{R}_{n+2}$-fundamental subset $B_{n+2} \subset B_{n+1}$ and an $\left(\mathcal{R}_{n+2}, B_{n+2}\right)$-splitting homeomorphism $h_{n+2}: I_{r_{n+2}} \times B_{n+2} \rightarrow B_{n+1}$. Take now any countable onto map $p_{n+2}$ from $B_{n+2}$ to a countable discrete set $D_{n+2}$ such that the diameter of the corresponding partition $\pi_{n+2}$ of $X_{0}$ is less then $\epsilon_{n+2}$, i.e. $(\triangle)$ is satisfied. 'Refining' $p_{n+2}$, if necessary, we can also assume that there exists an onto map $g_{n+2}: I_{r_{n+2}} \times D_{n+2} \rightarrow D_{n+1}$ such that the diagram

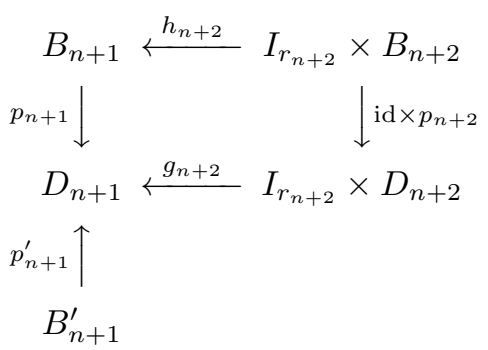

commutes and $(\nabla)$ holds for $n+2$. The diagram (2-4) is a 'reversed' version of (2-3) but with index $n+2$. Iterating this argument infinitely many times we come to (2-2). For that $(\nabla)$ is used: constructing (2-2) from 'measure preserving' diagrams (2-3) and (2-4) we obtain a fragment of (2-2) which is also measure preserving.

It should be noted that every time when we apply Lemmata 2.4 and 1.11 to define $B_{n+1}$ or $B_{n+1}^{\prime}$ we change the previously defined subsets $B_{1}, \ldots, B_{n}$ and $B_{1}^{\prime}, \ldots, B_{n}^{\prime}$. Indeed, we reduce them to some inessential reductions of $X$ and $X^{\prime}$. Making countably many steps we intersect countably many such inessential reductions to obtain $X_{0}$ and $X_{0}^{\prime}$ after all steps done.

\section{TYPE $I I I_{1}$}

In this section we prove Theorem 0.1 in the case when $T$ is of type $I I I_{1}$. We start with a crucial lemma which refines Lemma 1.4(ii) (cf. Lemma 2.3).

Lemma 3.1. Let $T$ be of type $I I I_{1}$ and let $A, B$ be two non-empty clopen subsets in $X$. Then for each $\epsilon>0$, there are open subsets $A^{\prime} \subset A, B^{\prime} \subset B$ and a homeomorphism $S: A^{\prime} \rightarrow B^{\prime}$ such that $\mu\left(A \backslash A^{\prime}\right)=\mu\left(B \backslash B^{\prime}\right)=0,(x, S x) \in \mathcal{R}_{T}$ for all $x \in A^{\prime}$, the map $A^{\prime} \ni x \rightarrow \rho_{\mu}(x, S x)$ is continuous and

$$
\left|\rho_{\mu}(x, S x)-\log (\mu(B) / \mu(A))\right|<\epsilon \text { for all } x \in A^{\prime} .
$$

Proof. We construct $S$ via an inductive process. Since the real $w:=\log (\mu(B) / \mu(A))$ belongs to $r\left(\rho_{\mu}\right)$, it follows from [Kr1], [HO] that there are $N>0$ and mutually disjoint Borel subsets $\left(A_{i}\right)_{|i|<N}$ of $A$ such that

(o) $1 / 2 \mu(A)<\sum_{|i|<N} \mu\left(A_{i}\right)<3 / 4 \mu(A)$,

(o) the sets $B_{i}:=T^{i} A_{i}$ are mutually disjoint Borel subsets of $B$,

(o) $\left|\rho_{\mu}\left(x, T^{i} x\right)-w\right|<\delta / 2$ for a very little $0<\delta<\epsilon$ at all $x \in A_{i}$ and

(०) $1 / 2 \mu(B)<\sum_{|i|<N} \mu\left(B_{i}\right)<3 / 4 \mu(B)$. 
Now find mutually disjoint clopen subsets $A_{i}^{0} \subset A$ such that

(о) $\mu\left(A_{i}^{0} \triangle A_{i}\right)<\delta \mu\left(A_{i}\right)$,

(०) the subsets $B_{i}^{0}:=T^{i} A_{i}^{0},|i|<N$, are mutually disjoint (clopen) subsets of $B$ and

(o) $\left|\rho_{\mu}\left(x, T^{i} x\right)-w\right|<\delta$ for all $x \in A_{i}^{0}$.

We now define a homeomorphism - a 'piece' of $S$-from a clopen subset $A_{0}:=$ $\bigsqcup_{|i|<N} A_{0}^{i}$ of $A$ onto a clopen subset $B_{0}:=\bigsqcup_{|i|<N} B_{0}^{i}$ by setting $S x:=T^{i} x$ if $x \in A_{i}^{0}$. Notice that

(o) $1 / 2<\mu\left(A_{0}\right) / \mu(A)<3 / 4,1 / 2<\mu\left(B_{0}\right) / \mu(B)<3 / 4$,

(o) $\left|\rho_{\mu}(x, S x)-w\right|<\delta<\epsilon$ for all $x \in A^{0}$, and

(o) the map $A_{0} \ni x \mapsto \rho_{\mu}(x, S x)$ is continuous.

This completes the first step of the inductive procedure. Now repeat the same for the pair of clopen subsets $A \backslash A_{0}$ and $B \backslash B_{0}$ (notice that the real $\log \left(\mu\left(B \backslash B_{0}\right) / \mu(A \backslash\right.$ $\left.A_{0}\right)$ ) belongs to $r\left(\rho_{\mu}\right)$ and it is very close to $\left.w\right)$ and so on. It remains to concatenate the pieces of $S$ that were constructed on all steps to obtain a homeomorphism $S$ from an open subset $A^{\prime}$ of $A, \mu\left(A^{\prime}\right)=\mu(A)$ to an open subset $B^{\prime}$ of $B, \mu\left(B^{\prime}\right)=\mu(B)$.

The following statement is a $I I I_{1}$-analogue of Lemma 2.4. We omit proof of it since it coincides almost verbally with that of Lemma 2.4 (just replace the reference to Lemma 3.1 with the reference to Lemma 2.3).

Lemma 3.2. Let $(\mu, T)$ be as in Lemma 3.1. Let $D, \widetilde{D}$ be two countable discrete spaces and $B$ a clopen subset of $X$. Let $\nu$ be a finite non-degenerated measure on $I_{r} \times \widetilde{D}$. Given $\epsilon>0$ and continuous onto maps $u: B \rightarrow D$ and $\widetilde{v}: I_{r} \times \widetilde{D} \rightarrow D$ such that $\mu \circ u^{-1}=\nu \circ \widetilde{v}^{-1}$, there are an $r$-uniform equivalence relation $\mathcal{S} \subset \mathcal{R}_{T}$ on $B$, an $\mathcal{S}$-fundamental subset $\widetilde{B} \subset B$, an $(\mathcal{S}, \widetilde{B})$-splitting homeomorphism $v: I_{r} \times \widetilde{B} \rightarrow B$ and a measure preserving continuous onto map $\widetilde{u}: \widetilde{B} \rightarrow \widetilde{D}$ such that the following diagram commutes

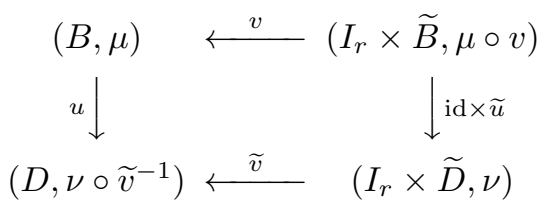

and $\left|\rho_{\mu}(v(j, \widetilde{b}), v(i, \widetilde{b}))-\log (\nu(j, \widetilde{u}(\widetilde{b})) / \nu(i, \widetilde{u}(\widetilde{b})))\right|<\epsilon$ for all $i, j \in I_{r}$ and $\widetilde{b} \in \widetilde{B}$. Here $\widetilde{B}$ and $\widetilde{D}$ are identified with the 'bottom' levels $\{0\} \times \widetilde{B}$ and $\{0\} \times \widetilde{D}$ of $I_{r} \times \widetilde{B}$ and $I_{r} \times \widetilde{D}$ respectively equipped with the induced measures.

We now prove the main result of this section.

Theorem 3.3. Let $(X, \tau, \mu, T)$ and $\left(X^{\prime}, \tau^{\prime}, \mu^{\prime}, T^{\prime}\right)$ be two dynamical systems of type $I I I_{1}$. They are almost continuously orbit equivalent.

Sketch of the proof. The structure of the proof is the same as in the proof of Theorem 2.5. We use the same notation as there and emphasize only the new ideas in the proof. Fix a sequence $\epsilon_{n}>0$ such that $\sum_{n=1}^{\infty} \epsilon_{n}<\infty$. As in the proof of Theorem 2.5, we will construct a commuting diagram (2-2). However the vertical arrows (and hence the orbit equivalence $\left(\phi^{\prime}\right)^{-1} \circ \phi$ ) will no longer be measure preserving. Nevertheless it will be nonsingular with continuous Radon-Nikodym derivative. 
We replace $(\nabla)$ with

$(\nabla)^{\prime}$ the Radon-Nikodym derivative on $\mathcal{R}_{n}$ is 'up to $\epsilon_{n}$ ' equivariant under $p_{n}$, i.e. given $d \in D_{n}$ and $i \in I_{r_{n}}$, the map $p_{n}^{-1}(d) \ni b \mapsto \rho_{\mu}\left(h_{n}(0, b), h_{n}(i, b)\right)$ is constant up to $\epsilon_{n}$

if $n$ is odd and

$(\nabla)^{\prime \prime}$ the Radon-Nikodym derivative on $\widetilde{\mathcal{R}}_{n}$ is up to $\epsilon_{n}$ equivariant under $\pi_{n}$, i.e. given $\left(i_{1}, \ldots, i_{n}, d_{n}\right) \in I_{r_{1}} \times \cdots \times I_{r_{n}} \times D_{n}$, the map

$$
p_{n}^{-1}\left(d_{n}\right) \ni b_{n} \mapsto \sum_{j=1}^{n} \rho_{\mu}\left(h_{j}\left(0, b_{j}\right), h_{j}\left(i_{j}, b_{j}\right)\right)
$$

is constant up to $\epsilon_{n}$, where $b_{j-1}:=h_{j}\left(i_{j}, b_{j}\right), j=n, n-1, \ldots, 2$,

if $n$ is even. We also replace references to Lemma 2.4 with references to Lemma 3.2. The rest of the construction is same.

Let us verify that $\mu \circ \phi^{-1} \sim \mu^{\prime} \circ \phi^{\prime-1}$ with continuous Radon-Nikodym derivative. First, we note that $\left(X_{0}, \mu\right)=$ proj $\lim _{n \rightarrow \infty}\left(X_{n}, \mu_{n}\right)$, where $X_{n}:=I_{r_{1}} \times \cdots \times I_{r_{n}} \times B_{n}$ and $\mu_{n}$ is the image of $\mu$ under the canonical homeomorphism of $X_{0}$ with $X_{n}$ (see the upper line in (2-2)). On the other hand, we have a sequence of measures $\nu_{n}$ on $I_{r_{n}} \times D_{n}$ 'intertwined' by the maps $g_{n}$. They lead naturally to an 'inverse' sequence of probability spaces

$$
\left(Z_{1}, \eta_{1}\right) \stackrel{\operatorname{id} \times g_{2}}{\longleftarrow}\left(Z_{2}, \eta_{2}\right) \stackrel{\operatorname{id} \times \operatorname{id} \times g_{3}}{\longleftarrow} \cdots,
$$

where $Z_{n}:=I_{r_{1}} \times \cdots \times I_{r_{n}} \times D_{n}$ (see the middle line in (2-2)). Let $\eta$ denote the inverse limit of $\left(\eta_{n}\right)_{n}$. This is a probability measure on $Z$. Notice that $\phi=$ proj $\lim _{n \rightarrow \infty} \phi_{n}$, where $\phi_{n}=\mathrm{id} \times \cdots \times$ id $\times p_{n}$ maps $X_{n}$ onto $Z_{n}$. We now verify that the sequence of continuous functions

$$
a_{n}: Z \ni z \mapsto a_{n}(z):=\log \left(d \mu_{n} \circ \phi_{n}^{-1} / d \eta_{n}\right)\left(z_{n}\right) \in \mathbb{R}
$$

converges uniformly on $Z$ along odd $n \rightarrow \infty$. For this, we introduce some notation. Given $i \in I_{r_{n}}$ and $d \in D_{n}$, we set $\nu_{n}(i \mid d):=\nu_{n}(i, d) / \nu_{n}(0, d)$. Then for all $i_{1} \in$ $I_{r_{1}}, \ldots, i_{n} \in I_{r_{n}}, d_{n} \in D_{n}$, we have

$$
\begin{aligned}
\eta_{n}\left(i_{1}, \ldots, i_{n}, d_{n}\right) & =\nu_{1}\left(i_{1} \mid d_{1}\right) \nu_{2}\left(i_{2} \mid d_{2}\right) \cdots \nu_{n-1}\left(i_{n-1} \mid d_{n-1}\right) \nu_{n}\left(i_{n}, d_{n}\right) \text { and } \\
\mu_{n} \circ \phi_{n}^{-1}\left(i_{1}, \ldots, i_{n}, d_{n}\right) & =\int_{p_{n}^{-1}\left(d_{n}\right)} \prod_{j=1}^{n} \exp \left(\rho_{\mu}\left(h_{j}\left(0, b_{j}\right), h_{j}\left(i_{j}, b_{j}\right)\right)\right) d \mu\left(b_{n}\right),
\end{aligned}
$$

where $d_{j-1}=g_{j}\left(i_{j}, d_{j}\right)$ and $b_{j-1}=h_{j}\left(i_{j}, b_{j}\right)$, for $j=n, n-1, \ldots, 2$. Since the map id $\times p_{n}$ is measure preserving, we deduce from $(\nabla)^{\prime}$ that

$$
\rho_{\mu}\left(h_{n}(0, b), h_{n}(i, b)\right)=\log \left(\nu_{n}(i \mid d)\right) \pm \epsilon_{n} \text { for all } b \in p_{n}^{-1}(d), i \in I_{r_{n}}, d \in D_{n},
$$

if $n$ is odd. On the other hand, $(\nabla)^{\prime \prime}$ yields

$$
\int_{A} \prod_{j=1}^{n} \exp \left(\rho_{\mu}\left(h_{j}\left(0, b_{j}\right), h_{j}\left(i_{j}, b_{j}\right)\right)\right) d \mu\left(b_{n}\right)=\frac{\mu \circ \phi_{n}^{-1}\left(i_{1}, \ldots, i_{n}, d_{n}\right) \mu(A)\left(1 \pm \epsilon_{n}\right)}{\mu\left(p_{n}^{-1}\left(d_{n}\right)\right)}
$$

for every $\left(i_{1}, \ldots, i_{n}, d_{n}\right) \in I_{r_{1}} \times \cdots \times I_{r_{n}} \times D_{n}$ and Borel subset $A \subset p_{n}^{-1}\left(d_{n}\right)$ if $n$ is even. This fact and (3-1) imply that $\left|a_{n+1}(z)-a_{n}(z)\right| \leq 2 \epsilon_{n}$ for all $z \in Z_{0}$ if $n$ is odd. Hence $\mu \circ \phi^{-1} \sim \eta$ with continuous Radon-Nikodym derivative. In a similar way one (changing the parity of $n$ ) can show that $\mu^{\prime} \circ \phi^{\prime-1} \sim \eta$ with continuous Radon-Nikodym derivative. 


\section{REFERENCES}

[CFW] A. Connes, J. Feldman and B. Weiss, An amenable equivalence relation is generated by a single transformation, Ergod. Th. \& Dynam. Sys. 1 (1981), 431-450.

[dJS] A. del Junco and A. Sahin, Dye's theorem in the almost continuous category, to appear in Israel J. Math..

[dJRW] A. del Junco, D. Rudolph and B. Weiss, Measure topological orbit and Kakutani equivalence, to appear in Disc. Cont. Dyn. Sys..

[Dy1] H. A. Dye, On groups of measure preserving transformations, I, Amer. Math. J. 81 (1959), 119-159.

[Dy2] H. A. Dye, On groups of measure preserving transformations, II, Amer. Math. J. 85 (1963), 551-576.

[Ep] I. Epstein, Orbit inequivalent actions of non-amenable groups, preprint.

[Hj] G. Hjorth, A converse to Dye's theorem, Trans. Amer. Math. Soc. 357 (2005), 30833103.

[HK] T. Hamachi and M. Keane, Finitary orbit equivalence of odometers, Bull. London Math. Soc. 38 (2006), 450-458.

[HKR] T. Hamachi, M. Keane and M. Roychowdhury, Finitary orbit equivalence and measured Bratteli diagrams, Colloq. Math. 110 (2008), 363-382.

[HO] T. Hamachi and M. Osikawa, Ergodic groups of automorphisms and Krieger's theorems, Seminar on Mathematical Sciences, 3. Keio University, Department of Mathematics, Yokohama, 1981. $113 \mathrm{pp}$.

$[\mathrm{KaW}]$ Y. Katznelson and B. Weiss, The classification of nonsingular actions, revisited, Ergodic Theory Dynam. Systems 11 (1991), 333-348.

[KS] M. Keane and M. Smorodinsky, Bernoulli schemes of the same entropy are finitarily isomorphic, Ann. Math. 109 (1979), 397-406.

[Kr1] W. Krieger, On non-singular transformations of a measure space. I, II, Z. Wahrscheinlichkeitstheorie und Verw. Gebiete 11 (1969), 83-97, and 98-119.

[Kr2] On ergodic flows and isomorphism of factors, Math. Ann. 223 (1976), 19-70.

[R1] M. Roychowdhury, $\left\{m_{n}\right\}$-odometer and the binary odometer are finitarily orbit equivalent, Ergodic theory and related fields, pp. 123-134, Contemp. Math., 430, Amer. Math. Soc., Providence, RI, 2007.

[R2] - Irrational rotation of the circle and the binary odometer are finitarily orbit equivalent, Publ. Res. Inst. Math. Sci. 43 (2007), 385-402.

[RRu] M. Roychowdhury and D. J. Rudoplh, Any two irreducible Markov chains are finitarily orbit equivalent, Isr. J. Math. (to appear).

[SWWr] D. Sullivan, B. Weiss and J. D. Wright, Generic dynamics and monotone complete $C^{*}-$ algebra, Trans. Amer. Math. Soc. 295 (1986), 795-809.

Institute for Low Temperature Physics \& Engineering of National Academy of Sciences of Ukraine, 47 Lenin Ave., Kharkov, 61164, UKRAine

E-mail address: danilenko@ilt.kharkov.ua

Department of Mathematics, University of Toronto, M5S 3G3, CANADA

E-mail address: deljunco@math.toronto.edu 